This is an author accepted manuscript version of this published paper:

Hannigan B. and Allen D. (2003) A tale of two studies: research governance issues arising from two ethnographic investigations into the organisation of health and social care. International Journal of Nursing Studies 40 (7) 685-695.

The published journal article can be found at: http://www.journalofnursingstudies.com/article/S0020-7489\%2802\%2900111-6/abstract

Please reference this paper using the full citation given above. 


\title{
A tale of two studies: research governance issues arising from two ethnographic investigations into the organisation of health and social care
}

\begin{abstract}
In a number of countries throughout the world attention is being paid to the ways in which health and social care research is undertaken and regulated. In the United Kingdom, new research governance frameworks are intended to promote improvement in research quality. This paper draws on our experiences of seeking research ethics committee approval for two investigations with the same research design, in order to address four governance issues: consistency within and between ethics committees; the assessment of vulnerable individuals regarding their suitability to participate in studies; the relationship between ethics and access; and ethics committee understanding of qualitative research.
\end{abstract}

\section{Keywords}

research governance; research ethics committees; vulnerable patients; informed consent; qualitative research. 


\section{Introduction}

Throughout the developed world increasing attention is being paid to the ways in which health and social care research is undertaken and regulated. In the United States, the Commission on Research Integrity was established in 1993 to tackle misconduct in biomedical and behavioural science (Commission on Research Integrity, 1995). Comparable measures have been taken in Europe. For example, the Danish Committee on Scientific Dishonesty published guidelines intended to promote high standards in the conduct of studies (Danish Committee on Scientific Dishonesty, 1998). The United Kingdom (UK) has recently witnessed the production of a number of new governance frameworks outlining the key responsibilities of all stakeholders involved in health and social care research (Department of Health, 2001a; Scottish Executive Health Department, 2001; Wales Office of Research and Development for Health and Social Care, 2001). Anecdotal evidence indicates that, in the UK certainly, attempts to strengthen the governance of health and social care research have been met with a mixed response. Some have expressed concern that the new governance arrangements will place unreasonable demands on researchers and that their over-enthusiastic application will result in unnecessary bureaucratic complexity that will compound existing demands on the research community. Others have countered that past experience indicates a clear need for robust regulatory frameworks in order to ensure that research subjects are protected and that health and social care research is of a high quality (Pirohamed, 2002).

In this paper we draw on our experiences of applying for research ethics committee (REC) approval for two studies with the same research design in order to explore a 
number of key research governance issues of significance to researchers, RECs and to organisations providing health and social care. These issues are: REC consistency; access to research participants and the assessment of vulnerable individuals regarding their fitness to participate in studies; the relationship between ethics and access to study sites; and research ethics committee understanding of qualitative methods.

\section{Research governance}

The UK research governance frameworks were developed against the background of a number of high profile cases of unacceptable research practice in the health and social care field. These include the case of the obstetrician Malcolm Pearce, who published a research report in the British Journal of Obstetrics and Gynaecology in which he falsely claimed to have carried out a novel surgical procedure and to have completed a randomised controlled trial (Dyer, 1995). More recently, the Royal Liverpool Children's Inquiry team uncovered evidence of the systematic removal of internal organs from the bodies of children, obtained without parental consent, ostensibly for the purposes of research into sudden infant death (Redfern et al., 2001).

The UK's research governance frameworks are comparable to other approaches to quality improvement and regulation in the health and social care field, such as the framework for clinical governance (Department of Health, 1998), in that they aim to raise the general standard of practice to the level of the 'leading edge'. The new frameworks are particularly intended to tackle the problem of lack of clarity regarding responsibility and accountability in the conduct of research. Key responsibilities are outlined for the range 
of stakeholders involved in health and social care research: health and social services providers; universities; research sponsors; ethical scrutiny committees; individual researchers; and users of services who participate in particular research projects (Department of Health, 2001a).

The Department of Health framework also re-asserts that the primary consideration in studies should be the dignity, rights, safety and well-being of research participants, and that National Health Service (NHS) research ethics committees have a key role to play in this area. A separate document issued by the Department of Health specifically addresses the role, responsibilities and working practices of RECs (Department of Health, 2001b). This document addresses the work of RECs in the light of international directives relating to health and social care research, and in the context of the new over-arching framework for research governance. The new governance arrangements for RECs confirm their status as independent bodies within the NHS, which exist to provide advice on the ethical dimensions of research projects that plan to involve users of health services, carers of health service patients, or NHS staff.

RECs operate within health authority boundaries, and within England and Wales have been subject to previous sets of guidance (Department of Health, 1991; 1997) which the new arrangements now supersede. Typically, the work of RECs has addressed a wide range of issues, including: the scientific basis of studies; the potential of studies to damage the health of participants; the likelihood and degree of distress and discomfort to participants; and the proposed procedures for obtaining and recording informed consent. 
In addition, it is usual for RECs to pay particular attention to research studies which aim to include vulnerable patients, including: children; women who may be pregnant; prisoners; and people with mental illnesses or learning disabilities (Tierney, 1995).

However, RECs have been criticised on a number of grounds, including: their medical dominance and lack of understanding of qualitative research studies (Dolan, 1999; Gelling, 1999; Stevenson and Beech, 1998); the lack of standardisation between committees in the ways in which they process applications (Redshaw et al., 1996); and the inconsistencies in outcome which can be encountered when identical or similar research proposals are scrutinised by different RECs (Nicholl, 2000). These issues present themselves as significant problems for health and social care researchers, and in this paper we describe our experiences of dealing with difficulties of this kind. Our aim is to draw conclusions for research governance and make recommendations for researchers, for ethics committees and for organisations which host research.

\section{The studies}

There is now widespread agreement within the nursing professions that in order to build a research base there is a need to move away from research centred on individuals in favour of a team approach focused on broad themes and/or programmes of work with strong research leadership (Department of Health, 1993; Emden, 1998; Higher Education Funding Council for England, 2001; Task Group 3, 2001; Norbeck, 1998; Wood, 1990). At the School of Nursing and Midwifery Studies at the University of Wales College of Medicine, UK, one such programme of research centres on the organisation and delivery 
of health and social care. The two studies which we draw on in this paper are part of this programme. The studies shared the same research design, and both aimed to explore how roles and responsibilities were managed in the provision of health and social care in order to identify those factors that helped and hindered integrated service provision.

Both studies employed a multiple case study, cross-site comparative design. Data were generated using established ethnographic methods. Study 1 focused on adults undergoing stroke rehabilitation, who, in the opinion of service providers, had complex continuing care needs. The study was undertaken in two Welsh health authorities. Four ethnographic case studies were carried out in each site centred on the client and their surrounding web of care. Each case was followed for six months as the patient progressed from the acute sector to home. Snowball sampling was used to identify the key players and processes involved in the planning and provision of services in each case. Interviews were carried out with clients and their significant others, and with service providers involved in their care and with other key personnel. Critical events, such as case conferences and home visits, were observed and in some instances tape-recorded. Case notes and additional documentation were also consulted. These data were used to build up a comprehensive picture of each case in which their care was mapped. Data handling was supported by the computer-assisted qualitative data analysis software programme, Atlas.ti (Scientific Software Development/Scolari, 1997).

Study 2 replicates the design of study 1 and focuses on service provision to people with severe mental health problems. This work is on-going. Owing to resource constraints 
only six cases have been studied for four to five months each. The delivery of care to clients of two NHS trusts and two local authority social services departments is being compared. The decision to replicate the design of study 1 in the second study was a strategic one, and was linked to the development of research capacity. Replication studies are valuable in that they permit findings to be compared and contrasted, and encourage the development of methodological expertise within research units. There are also practical benefits as protocols and templates can be shared; both the information sheets produced for study 2, and applications submitted for REC approval, drew heavily on documents produced in study 1 .

\section{Applying for ethical approval}

\section{Study 1}

In study 1 it was necessary to apply to two RECs because the research was being carried out in two health authorities. An application template was developed and the information incorporated into the different formats required for the two committees. In both cases REC approval was granted in a straightforward way. However, despite the fact that it drew heavily on the research design used in study 1 and utilised the template for the REC application form, the experience of obtaining REC approval in study 2 proved to be very different. In the following sections we describe this process, drawing out the significant points of comparison with study 1 and the broader issues that are raised for research governance.

\section{Study 2}


Although study 2 adopted the research design developed in study 1, the project differed significantly from the first in that it planned to include people with mental health difficulties. This raised specific, and important, ethical issues. It is usual to regard people experiencing mental health problems as being particularly vulnerable research participants (Koivisto et al., 2001). People with major mental illnesses such as schizophrenia are often considered to lack the capacity needed to give their informed consent to participate in research studies. 'Capacity' in this context refers to the ability of individuals to exercise autonomy and self-determination (Drane, 1984). In a recent UK report, the individual who is 'without capacity' is one who is judged to be:

1. unable by reason of mental disability to make a decision on the matter in question, or 2. unable to communicate a decision on that matter because he or she is unconscious or for any other reason.

(Law Commission, 1995)

'Mental disability' in this context is defined as "a disability or disorder of the mind or brain, whether permanent or temporary, which results in an impairment or disturbance of mental functioning" (Law Commission, 1995).

One approach to the protection of people with mental illness is to exclude all those with “impairment or disturbance of mental functioning" from participating in research studies. However, this approach would lead to the unnecessary protection of individuals who, whilst experiencing some degree of mental impairment or disturbance, also possess the 
ability to give consent (Usher and Holmes, 1997). Many people with mental health problems might also object to being automatically excluded from research studies, and fear that not having the choice of participating would lead to their views and experiences remaining unknown. Finally, it is important to note that an individual's 'capacity' is not an absolute. The ability to exercise autonomy and to make informed decisions - for all people, and not just those with mental health difficulties - is liable to fluctuation.

An additional ethical dimension was raised by the decision to include in the research sample people whose care and treatment was organised under sections of the Mental Health Act (1983) for England and Wales. The Act establishes the conditions under, and the procedures through, which people with mental disorders can be compulsorily admitted to hospital for the purposes of assessment and treatment. The Act also sets out the broad principles for the provision of 'aftercare'. In addition, an amendment, the Mental Health (Patients in the Community) Act (1995), brought in a new power of 'supervised discharge'. This meant that individuals previously detained in hospital under certain treatment sections of the 1983 Act could, in some circumstances, be obliged to reside in a specified place, attend specified venues for treatment or other activities, and allow access to named mental health professionals.

The inclusion in research studies of people subject to sections of the Mental Health Act needs to be done with extreme care. People whose care and treatment is organised under sections of the Act are under a form of constraint. This places an additional obligation on researchers to ensure that those who participate in studies give 'true' informed consent 
(Wing, 1999). Guidance on the inclusion in studies of detained patients does exist, but seems contradictory. Local guidance produced by the REC in the area in which study 2 is taking place includes a document addressing research involving patients unable to give informed consent. Included in this document is a section which indicates that people detained under the Mental Health Act "should not participate in research in normal circumstances". The document does not consider the possibility of detained patients being able to give informed consent. In contrast to this local guidance, the position of the body charged with overseeing the operation of the Mental Health Act and of protecting the rights of detained patients in England and Wales is that people subject to sections of the Act should be able to participate in both 'therapeutic' and 'non-therapeutic' research, subject to certain conditions being met (Mental Health Act Commission, 1997). In the case of 'non-therapeutic' research, which includes research like study 2 , the Commission states that "the position in law for the detained patient is the same as for other citizens" (Mental Health Act Commission, 1997, p6, emphasis in original). These issues are discussed in more detail below.

The initial application for REC approval in the second study stressed awareness of the range of ethical issues raised by the proposal. Criteria for the selection of case study subjects were also included, as Figure 1 shows.

[insert Figure 1 about here]

This initial REC application was unsuccessful. Included in Figure 2 are extracts from the 
committee's response.

\section{[insert Figure 2 about here]}

As Figure 2 indicates, the REC had a number of concerns with the application for ethical approval. First, the committee expressed the view that the objectives in study 2 could not be met. Yet as we have indicated, in study 1 two successful REC applications were made which described the same objectives and the same research methods. The committee did not explain why it believed the objectives in study 2 could not be met, and so the reasons for its decision remain opaque. However, this does raise the issue of consistency in research governance processes ( $c f$. Nicholl, 2000).

One possible explanation for the inconsistency between committees could have been their differing degrees of understanding of qualitative methods, and of the value of undertaking qualitative studies. There is literature indicating that RECs sometimes fail to understand research which proposes to use non-quantitative methods ( $c f$. Dolan, 1999; Gelling, 1999; Stevenson and Beech, 1998). Dolan (1999) has argued that limited knowledge of qualitative research by RECs presents itself as a particular problem for nurse researchers, who may be more likely than other health researchers to design studies which use nonquantitative methods. Dolan has also questioned the legitimacy of RECs in objecting to the methodological basis of studies rather than focusing exclusively on the ethical dimensions. 
Other factors may have been important in determining the different REC responses to the two studies. Although the studies had the same design, they also had differences which may have helped to shape the committees' decisions. At the time the REC applications were made, study 1 had already received external research funding and this may have functioned to afford it a legitimacy in the eyes of the committees compared to study 2 , which at that point had no external funding. Moreover, it was a requirement of the REC application forms to state whether the research was being undertaking as part of a programme of study. Whilst study 1 was undertaken by an experienced research team, study 2 is being carried out for the purposes of a $\mathrm{PhD}$, and this may have resulted in the committee taking a more critical stance. REC decision-making processes inevitably take place in a social context, and members deploy a number of processes in order to find meaning in a given situation (Garfinkel, 1967). Although we cannot say with any certainty how significant these key differences between the two studies were in practice, it has raised our awareness of the subtle clues that are contained in application forms, and has heightened our sensitivity to issues of interpretation and presentation.

The resubmitted application for ethical approval in study 2 was designed to address the committee's concerns (Figure 3). The study objectives were condensed with the aim of improving clarity, and the relationship between study 1 and study 2 was more strongly emphasised. In addition, the long tradition of ethnographic research and its capacity to generate important data associated with the provision of health and social care was underlined. 
The second objection raised by the REC related to issues of access. The REC criticised the application for having insufficient detail about service providers who would be involved in the study. It also noted that it was unclear who would assess the 'fitness' of patients to participate in the project. These objections raise further research governance issues. First, is the relationship between seeking REC approval and negotiating access to study sites. In study 2 , the view of the ethics committee appeared to be that access negotiations should have started before an application was made for ethical approval. However, as later experience in study 2 demonstrated, one of the first questions which health and social care 'gatekeepers' typically ask during access negotiations in research sites is whether formal ethical approval has been obtained. In study 1 it was a requirement of the application for research funding that key research collaborators were identified. Key service managers, senior clinicians and policy makers agreed to support the study and to function as a steering group. However, although all had good service links, none were in a position to grant direct access to the study sites in which the research was taken forward. These processes were put in train once REC approval had been secured. Nevertheless, the identification of key service collaborators on the REC application appears to have satisfied both RECs and raises the question of how well 'research access' is understood by ethics committees.

The REC's response in study 2 also raises important questions regarding the control of research access to health service users. The committee in this case stated that only a medical practitioner could assess the capacity of individual patients to give informed consent. This statement granted a powerful research 'gatekeeping' role to members of the 
medical profession (Service Users' Experiences Research Group, 2000), which appears to run counter to current trends in health and social care delivery in the UK. In the contemporary NHS, the importance of partnership between the professions, and between the professions and users of their services, is increasingly recognised (Department of Health, 1998). It therefore appears out-of-step for an ethics committee to preserve for doctors alone the responsibility for assessing the suitability of people to take part in research studies. We return to this issue at a later point below.

In response to the REC's concerns in study 2 access negotiations with key individuals in each of the two selected study sites were brought forward. As in study 1, the process of negotiating access was complex, involving contact with multiple gatekeepers. An important task was to secure letters of support from senior professionals, which were later attached to the resubmitted REC application as evidence of collaboration. Over the course of these negotiations the interdependence of negotiating research access and achieving ethical approval was explicitly discussed with the study participants, who became partners in the process of securing the REC's agreement that study 2 could proceed.

Considerable work was also undertaken to address the REC's concerns regarding the possible inclusion in study 2 of people subject to sections of the Mental Health Act, and to address the concerns regarding the assessment of suitability to take part in the study. As Figure 2 shows, the committee stated that it was not clear whether liaison regarding the fitness of detained patients to participate would take place with responsible medical practitioners, or with "medical officers appointed under the terms of the Mental Health 
Act". The REC's suggestion that it might be necessary to obtain the opinion of independent doctors regarding the suitability of people subject to sections of the Act to take part in study 2 appears to run contrary to the position of the Mental Health Act Commission (MHAC), as we have outlined above. The second REC application (Figure 3) included information directly extracted from the MHAC's position paper on research involving detained patients (Mental Health Act Commission, 1997). Material was also included from the current Mental Health Act Code of Practice (Department of Health and Welsh Office, 1999). The information from these two sources addressed a number of important issues relating to mental illness, capacity and research participation. The new application for ethical approval in study 2 also included explicit information regarding the selection of service users to take part in the study, the assessment of their suitability, and the processes through which they would be recruited.

[insert Figure 3 about here]

\section{Unexpected outcomes}

This new REC application for study 2 was submitted to the same panel that had scrutinised, and rejected, the first application. The panel's response was most unexpected. The committee wrote that it had "....agreed that your proposal was a survey and as such was outside the remit of the research ethics committee". This was a surprising outcome, given the range of questions raised by the panel in its first deliberation. Many of the issues raised in the first application had been addressed in the resubmitted proposal. Nonetheless, it was still not expected to hear that the planned research was, from the 
REC's perspective, either not 'research' at all, or not 'research' which required REC approval.

At a pragmatic level this decision meant that the study could proceed. Data generation has now been completed in both selected study sites, and data analysis is well underway. The approach taken to the recruitment of case study subjects followed the procedure detailed in the resubmitted REC application, and reproduced here in Figure 3. However, the refusal of the REC which scrutinised the second application for ethical approval to recognise this research as 'research' again raises important governance concerns. Once more the issue of consistency is raised. In the literature, inconsistency has most often been reported as being a particular problem in relation to multi-site studies, where approval for a study in one area has sometimes been matched by extended delay or refusal in another (While, 1995). As an approach to addressing this, multicentre research ethics committees (MRECs) were introduced in the UK in order to simplify the process of obtaining ethical approval for studies taking place in multiple study sites (Alberti, 2000), although it is not clear that the problem of variation between committees has been helped by these changes (Tully et al., 2000). However, as our experiences show, inconsistency can take a number of different forms: in this case in the form of inconsistency by a single REC over time. A second governance issue raised by the REC's decision on the resubmitted application for approval in study 2 is, again, that of a lack of understanding of qualitative research studies. Lack of understanding of this sort may have contributed to the REC's decision to designate study 2 as a 'survey', and not as 'research'. 


\section{Conclusions and recommendations}

This paper has drawn on our experiences of seeking research ethics committee approval for two investigations which shared the same research design in order to address key research governance issues. In this final section we draw conclusions from our experiences, and make recommendations for researchers, ethics committees and organisations which host research. We also consider the implications of our findings for the implementation of the new Department of Health research governance framework.

Our experiences suggest that there are important lessons for all parties in relation to consistency. First, it is important for researchers preparing applications for ethical approval for replication studies to guard against complacency. Additional and specific ethical issues can be raised when a research design and methods used in one context are transferred for use in another. However, our experiences bear out that even when care is taken to consider these additional ethical issues, research ethics committees are still liable to make unexpected decisions.

REC inconsistency can partly be explained by the nature of the work that they engage in. Ethical decision-making is a complex process, and one that is not amenable to the application of formulaic guidelines (Dingwall, 1980). Given this, it is to be expected that different committees will sometimes reach different conclusions when faced with the same study. It is not clear how far the new UK research governance frameworks will help to reduce the unpredictability of REC decision-making. Guidance from the Department of 
Health urges committees to act in a reasonable, competent and timely manner, and to make clear to researchers their reasons for rejecting studies (Department of Health, 2001b). The new arrangements for RECs also emphasise their independence. Whilst not compromising this independence, we consider it important for ethics committees to consider the principle of precedent in their deliberations, and through doing this to promote as far as possible a consistent approach towards making decisions.

Demonstrating consistency in this way is one means through which RECs can meet their responsibilities towards researchers (Department of Health, 2001b). Researchers, for their part, need to be sensitive to the interpretative work that inevitably goes on in REC decision-making, and ensure that the information contained in their applications is unequivocal.

Our experiences have also revealed evidence of a continued bias, in at least some RECs, towards biomedical science and the medical profession. This was shown in the limited understanding of qualitative research demonstrated by the REC which scrutinised study 2. Recent efforts have been made to raise awareness within the medical profession of the contribution which qualitative studies can make (see, for example, Pope and Mays, 1995). However, qualitative researchers often still need to engage in a process of 'translation' when presenting their work to bodies which, like RECs, are more familiar with experimental and other quantitative approaches. We suggest that this process of translation could usefully include highlighting the similarities between qualitative and quantitative research, rather than emphasising their putative differences. It may be better, for example, for qualitative researchers completing applications for REC approval to 
emphasise the degree of 'fit' between their research objectives and their chosen methods, rather than to engage in lengthy epistemological excursions. This problem of limited understanding of qualitative methods may also be helped by the adoption of recommendations made in the UK's new research governance framework. Notably, the Department of Health states that RECs are required to include expert members with "relevant methodological and ethical expertise in qualitative or other research methods applicable to health services, social science and social care research" (Department of Health, 2001b, p13).

With respect to the issue of the assessment of the fitness of individuals to participate in studies, the UK's new governance arrangements do not appear to favour the views of one professional group over another. Principal researchers have the responsibility, "when a study involves participants under the care of a doctor, nurse or social worker for the condition to which the study relates", to inform those professionals that their patients are being invited to participate (Department of Health, 2001a, p25). Care professionals, for their part, are required to satisfy themselves before agreeing for researchers to approach their patients or clients that the appropriate scrutiny of the proposed research has taken place. The new guidelines for RECs indicate that committees should pay attention to the processes through which research participants are recruited, but do not indicate any particular responsibilities for any individual professional group (Department of Health, 2001b). Our view is that the assessment of the suitability of a patient or service user to participate should take account of a range of factors, including the patient's condition and their social circumstances, the nature and degree of health and social care professional 
involvement, and the nature of the proposed research. In some instances, the initial assessment of an individual's suitability to take part in a study might most appropriately fall to a single practitioner, of any discipline, who is best acquainted with the individual. For example, in study 2 the assessment of the suitability of individuals who were not detained under sections of the Mental Health Act to take part in the project could initially have been undertaken by the potential case study subject's 'key worker' or 'case manager'.

In this paper we have also shown that the relationship between ethics and access can be a complex one. After the rejection of the first application to the REC in study 2, the processes of applying for ethical approval and negotiating access to study sites became closely interwoven. The Department of Health now states that the "ethical review by the REC should occur in parallel with the consideration of the proposed research by NHS host organisations" (Department of Health, 2001b, p17). At the end of this process, provider organisations must be satisfied that all research conducted within their boundaries has been approved by an appropriate ethics committee (Department of Health, 2001a), and RECs must be satisfied of the 'adequacy' of study sites for the research proposed (Department of Health, 2001b). There are further lessons for all parties here. Our experiences, and the recent guidance from the Department of Health, suggest that from the outset researchers should work towards ethical approval and access to study sites simultaneously, as far as this is possible. For gatekeepers in health and social care organisations hosting research, it is therefore important to remember that ethical approval will often not be granted until relatively advanced research access negotiations have been 
concluded.

We have highlighted ways in which the adoption of the Department of Health's new research governance framework may help to reduce in the UK the kind of difficulties which we encountered in study 2 . However, it is salutary to note that UK RECs historically have a poor record in adhering to published guidelines (see, for example, Gilbert et al., 1989; Tully et al., 2000). As we observed earlier in this paper, it will never be possible, or appropriate, for the ethical dimensions of research studies to be considered in a prescriptive manner. In the future, therefore, researchers are likely to continue to encounter difficulties similar to ours, although it is to be hoped that the incidence of these will lessen. Ultimately, the larger question raised by our paper is: where does the responsibility to ensure that studies are ethically sound lie? The answer is as it always has been: with researchers. Preparing research proposals for formal ethical scrutiny is an important exercise in obliging researchers to think critically about the ethical dimensions of their studies. Whatever the outcome of an application to a REC, however, the ethical dilemmas raised in the day-to-day undertaking of a research project can only be addressed by the researcher or the research team.

\section{Acknowledgements}

Study 1 was partially supported through a grant from the Wales Office of Research and Development for Health and Social Care, and study 2 was partially supported through the award of a Nursing Research Fellowship from the Smith and Nephew Foundation. 


\section{References}

Alberti, K.G.M.M., 2000. Multicentre research ethics committees: has the cure been worse than the disease? British Medical Journal 320, 1157-1158.

Allen, D., Griffiths, L., Lyne, P., Monaghan, L., Murphy, D., 2000. Delivering health and social care: changing roles, responsibilities and relationships. Cardiff and Swansea: School of Nursing and Midwifery Studies, University of Wales College of Medicine/School of Health Science, University of Wales (Swansea) [Final report to Wales Office of Research and Development for Health and Social Care, Cardiff].

Commission on Research Integrity, 1995. Integrity and misconduct in research: report of the Commission on Research Integrity. Report to the Secretary of Health and Human Services, the House Committee on Commerce and the Senate Committee on Labor and Human Resources. US Department of Health and Human Services, Rockville.

Danish Committee on Scientific Dishonesty, 1998. Guidelines for good scientific practice. Danish Committee on Scientific Dishonesty, Copenhagen.

Department of Health, 1991. Local Research Ethics Committees (HSG(91)5). Department of Health, London.

Department of Health, 1993. Report of the taskforce on the strategy for research in nursing, midwifery and health visiting. HMSO, London. 
Department of Health, 1997. Ethics committee review of multi-centre research (HSG(97)23). Department of Health, London.

Department of Health, 1998. A first class service: quality in the new NHS. Department of Health, London.

Department of Health, 2001a. Research governance framework for health and social care. Department of Health, London.

Department of Health, 2001b. Governance arrangements for NHS research ethics committees. Department of Health, London.

Department of Health and Welsh Office, 1999. Code of practice: Mental Health Act (1983). The Stationery Office, London.

Dingwall, R., 1980. Ethics and ethnography. Sociological Review 28 (4), 871-91.

Dolan, B., 1999. Guest editorial: the impact of local research ethics committees on the development of nursing knowledge. Journal of Advanced Nursing 30 (5), 1009-1010.

Drane, J.F., 1984. Competency to give an informed consent: a model for making clinical assessments. Journal of the American Medical Association 252, 925-927. 
Dyer, O., 1995. Consultant struck off for fraudulent claims. British Medical Journal 310, $1554-1555$.

Emden, C., 1998. Establishing a 'track record': research productivity and nursing academe. Australian Journal of Nursing 16, 29-33.

Garfinkel, H., 1967. Studies in ethnomethodology. Prentice Hall, Englewood Cliffs NJ.

Gelling, L., 1999. Role of the research ethics committee. Nurse Education Today 19, 564569.

Gilbert, C., Fulford, K.W.M., Parker, C., 1989. Diversity in the practice of district ethics committees. British Medical Journal 299,1437-9.

Higher Education Funding Council for England, 2001. Research in nursing and allied health professions. Report of the Task Group 3 to HEFCE and the Department of Health. Higher Education Funding Council for England, London.

Koivisto, K., Janhonen, S., Latvala, E., Väisänen, L., 2001. Applying ethical guidelines in nursing research on people with mental illness. Nursing Ethics 8, 328-339. 
Law Commission, 1995. Report number 231: Mental incapacity. The Stationery Office, London.

Mental Health Act Commission, 1997. Position Paper 1: Research involving detained patients. Mental Health Act Commission, Nottingham.

Nicholl, J., 2000. The ethics of research ethics committees. British Medical Journal 320, 1217.

Norbeck, J.S., 1998. Teaching, research and service: striking the balance in doctoral education. Journal of Professional Nursing 14, 197-205.

Pirohamed, J., 2002 (April). Informed consent in clinical research - are we doing it properly? Paper presented at the Annual RCN Research Society Conference, University of Exeter, Exeter.

Pope, C., Mays, N., 1995. Qualitative research: reaching the parts other methods cannot reach: an introduction to qualitative methods in health and health services research. British Medical Journal 311, 42-45.

Redfern, M., Keeling, J., Powell, E., 2001. The Report of The Royal Liverpool Children's Inquiry. Report to the House of Commons, London. 
Redshaw, M.A., Harris, A., Baum, J.D., 1996. Research ethics committee audit:

differences between committees. Journal of Medical Ethics 22, 78-82.

Scientific Software Development/Scolari, 1997. Atlas.ti: the knowledge workbench. Scientific Software Development/Scolari, Berlin/London.

Scottish Executive Health Department, 2001. Research governance framework for health and community care. Scottish Executive, Edinburgh.

The Service Users' Experiences Research Group, School of Nursing and Midwifery Studies, University of Wales College of Medicine, 2000. User involvement in userfocused research? [Working Paper 1]. Nursing, Health and Social Care Research Centre, School of Nursing and Midwifery Studies, University of Wales College of Medicine, Cardiff.

Stevenson, C., Beech, I., 1998. Playing the power game for qualitative researchers: the possibility of a post-modern approach. Journal of Advanced Nursing 27, 790-797.

Task Group 3, 2001. Promoting research in nursing and the allied health professions: a report to task group 3 by the CPNR, CHEMS Consulting, the Higher Education Consultancy Group and the Research Forum for Allied Health Professions. Higher Education Funding Council for England, London. 
Tierney, A., 1995. The role of research ethics committees. Nurse Researcher 3, 43-52.

Tully, J., Ninis, N., Booy, R., Viner, R., 2000. The new system of review by multicentre research ethics committees: prospective study. British Medical Journal 320, 1179-1182.

Usher, K., Holmes, C., 1997. Ethical aspects of phenomenological research with mentally ill people. Nursing Ethics 4, 49-56.

Wales Office of Research and Development for Health and Social Care, 2001. Research governance framework for health and social care in Wales. Wales Office of Research and Development for Health and Social Care, Cardiff.

While, A., 1995. Ethics committees: impediments to research or guardians of ethical standards? British Medical Journal 311, 661.

Wing, J., 1999. Ethics and psychiatric research. In: Bloch, S., Chodoff, P., Green, S.A. (Eds.), Psychiatric ethics ( $3^{\text {rd }}$ edn.) Oxford University Press, Oxford, pp.461-477.

Wood, F., 1990. Factors influencing research performance of university academic staff. Higher Education 19, 81-100. 


\section{Figure 1}

\section{Extracts from first application for research ethics committee approval in study 2}

\section{Study objectives:}

1. map the network of health and social care providers involved in the delivery of services to a series of case study subjects $(n=4-8)$ over a period of up to four months each;

2. locate the research findings within the broader policy context at both local and national level;

3. undertake detailed study of the ways in which carers manage their respective roles and responsibilities in the delivery of health and social services;

4. identify the range of factors related to interagency collaboration which, in the opinion of local stakeholders, contribute to or detract from the effectiveness and quality of service provision in the study settings;

5. feed back the findings to the study settings, enabling critical reflection on the delivery of care and assisting in future service planning;

6. use these findings to make recommendations concerning the development of roles and responsibilities in the provision of community mental health care;

7. use these findings as a starting point for further studies;

8. develop the mapping exercise as a method for increasing understanding of the complex interfaces involved in the delivery of community mental health care;

9. share and disseminate the research findings to a multidisciplinary audience;

10. produce, as the end result of a comprehensive research training, a $\mathrm{PhD}$-level thesis.

The aims and objectives of this study determine that access be gained to individuals whose difficulties are both severe and long-lasting. Access may, therefore, be sought to individuals who are subject to sections of the Mental Health Act (1983) at their point of entry into the study. Access may also be sought to individuals who remain subject to formal care and treatment throughout the duration of their participation in the project. This would include, for example, the case of an individual discharged from hospital to home under section 25 of the Mental Health (Patients in the Community) Act (1995). Finally, there is the possibility that some individuals may become subject to the provisions of the Act during their participation in the study.

In seeking ethical approval to include people detained under the Act in this study, the following criteria for their participation are offered. First, no individuals suffering from an acute episode of mental illness will be approached in order to request their participation in the project. Hospital inpatients detained under the Act will only be approached as they are preparing for discharge into the community. Second, in the case of individuals subject to sections of the Act and approaching hospital discharge, the assent of the nearest relative and/or significant other will be sought. This will be in addition to the seeking of the informed consent of the service user him/herself. Third, where an individual participating in the study becomes acutely ill and is admitted to hospital, either informally or, particularly, under a section of the Mental Health Act, the assent of the nearest relative and/or significant other to the continued following of the service user's care will be sought. In all instances, the assent of appropriate clinicians, the 
Responsible Medical Officer [consultant psychiatrist] included, will also be sought. No extra demands will be made on either service users or their informal carers during critical periods, such as those associated with admission to hospital. Practitioners will also be asked, where appropriate, to give only retrospective accounts of their work during the period after caring for an acutely ill case study subject.

It is, finally, anticipated that the background of the principal researcher as a qualified and experienced community mental health nurse will be invaluable in ensuring that the clinical needs of case study subjects remain paramount, and in ensuring that professional judgement and sensitivity is maintained throughout the period in which all service users participate in the study. 


\section{Figure 2 \\ Extracts of response from research ethics committee to first application for approval to undertake study 2}

The Panel had a number of specific concerns but in general did not believe from the information provided that the study objectives could be met.

The Panel were concerned over the apparent lack of involvement of anyone from the NHS Trust. The Panel were unclear who would decide whether or not a patient was fit to take part in this study. Whilst you intend to include only patients able to give informed consent, the medical staff responsible for these patients are the sole parties to state whether or not the patients would be capable of giving informed consent. The Panel were concerned that the application gave no details of whether or not you intended to approach the medical staff responsible for these patients, or whether you intended to liaise with medical officers appointed under the terms of the Mental Health Act. 


\section{Figure 3 \\ Extracts from second application for research ethics committee approval in study 2}

The final report arising from [study 1] has been favourably received, and has demonstrated the value of in-depth qualitative research methods as a means of generating answers to important questions associated with the provision of health and social care.

\section{Principal objectives for this study are to:}

1. investigate the ways in which health and social care professionals manage their respective roles and responsibilities in the delivery of services to eight case study subjects with severe mental health problems;

2. map the network of health and social care providers involved in each of the case study subjects over a four month period;

3. identify the factors which, in the opinion of local stakeholders, contribute to or detract from the effectiveness of interagency and interprofessional collaboration in the study settings;

4. locate the research findings within the local and national policy context.

Subjects will be purposively selected in consultation with the responsible consultant psychiatrists and with other health and social care professionals. One criterion for subject selection will be the responsible consultant's assessment of the capacity of identified clients to give informed consent. The enclosed letters from [two consultant psychiatrists] indicate that they have agreed to take on this responsibility. Once potential subjects have been identified, the initial invitation to participate will be made on behalf of the principal researcher by appropriately-placed practitioners.

The aims and objectives of the study determine that access may be sought to individuals who are subject to sections of the Mental Health Act (1983). How care is delivered to people whose care and treatment is organised under sections of the Act is of particular analytic interest. Providing aftercare services under section 117, for example, is a critical test of the ability of different professionals and agencies to collaborate effectively. It is of equal analytic interest to gain an understanding of how well services work together when sections of the Mental Health Act are first applied for, and when individuals are formally admitted to hospital. It is also possible that some of the selected subjects may experience episodes of acute mental illness during their participation in the study.

These issues raise important ethical concerns. In the most recent edition of the Mental Health Act Code of Practice (Department of Health and Welsh Office 1999), it is stated that "mental disorder does not necessarily make a patient incapable of giving or refusing consent" (section 15.12). In its position paper on research involving patients detained under the Mental Health Act (1983), moreover, the Mental Health Act Commission (1997) has proposed that, "if a patient has capacity to consent to participation in research, and does in fact give actual and informed consent, then participation should not be prevented unless (a) involvement conflicts with any provision of the 1983 Act; (b) involvement is inconsistent with treatment being received as a detained patient". 
In seeking REC approval to include in this study people detained under the Mental Health Act (1983), and to include people who may experience episodes of acute mental illness during the period of their participation, the following criteria are offered:

- patients approached to participate in the study will only include those who have been assessed by the responsible consultant psychiatrist as having capacity to consent; only patients who have capacity to consent and who actually give informed consent will be included; patients will only enter the study with the explicit agreement of the responsible consultant; no individuals suffering from an acute episode of mental illness will be approached in order to request their participation in the study;

- the agreement of the responsible consultant to the continued participation of case study subjects who become acutely ill will be obtained;

- where an individual participating in the study becomes acutely ill, the assent of the nearest relative and/or significant other to the continued following of the service user's care will be sought;

- following guidance from the Mental Health Act Commission (1997), the Approved Social Worker (ASW) and other involved mental health professionals working with detained patients will be consulted prior to an approach being made to the patients to participate in the study;

- hospital inpatients detained under the Act will only be approached to participate as they are preparing for discharge into the community;

- in the case of individuals subject to sections of the Act and approaching hospital discharge, in addition to the obtaining of the patient's informed consent the assent of the nearest relative and/or significant other will be sought, subject to the patient's agreement to this approach being made.

Throughout this study, the background of the principal researcher as a qualified and experienced community mental health nurse will be invaluable in ensuring that the clinical needs of case study subjects remain paramount, and in ensuring that professional judgement and sensitivity is maintained. 